\title{
Systems medicine in oncology
}

\author{
José Costa
}

For the first time, advances in a wide diversity of fields make it feasible to investigate diseases as bio-systems via an integrative approach that includes clinical examinations, experimental modeling and in-silico simulation. Systems medicine seeks to understand perturbed physiological systems and complex pathologies in their entirety by integrating all levels of quantitative functional, structural and morphological information into a coherent model. In contrast to systems biology, systems medicine is geared towards obtaining clinical impact with both diagnostic and therapeutic end points.

The reduction of systems medicine to medical practice requires the integration of clinical data, in vivo imaging, ex vivo morphology and comprehensive data sets. Systems medicine requires knowledge, engineering and the use of artificial intelligence. Multiscale in-silico modeling is central to achieving this aim because it bridges the divide between molecular (i.e. genomics, proteomics, metabolomics) and macroscopic imaging in an effort to understand, diagnose and target diseases as complex dynamic systems.

Early examples of integrating information to increase the predictive power of morphological tests are beginning to yield promising results (Costa J Arch Pathol Lab Med, in press). Improvements in biological sample analysis and the instruments and technologies for imaging will allow real-time data sets to be obtained from patients. Systems medicine can use existing resources, creating trans-disciplinary teams of physicians, engineers, and scientists such that their associated competencies and skills are tailored to address the nature of the problem to be solved.

Ideally, systems medicine integrates seven approaches, each of which requires specific expertise. The first approach comprises disease-focused units that combine clinical expertise with conceptual insight into medical problems. These units identify the necessary patient population and build high quality
The

capabilities of

each and every

component

of systems

medicine

are sharply

focused on a

specific clinical

problem.

J Costa is an Advisory

Board member of

Nature Clinical

Practice Oncology.

\section{Competing interests}

The author declared no

competing interests.

www.nature.com/clinicalpractice doi:10.1038/ncponc1070 bio-repositories linked to clinical databases. Second, quantitative analytical sciences, mathematical modeling and computational biology are central to data acquisition, mining and display, biophysical analyses, and multiscale in-silico modeling. Third, diagnostic imaging, nuclear medicine, and functional imaging collectively provide digitized high-density macroanatomical and functional data sets. Fourth, analytical laboratory technologies enable multimodality and quantitative interrogation of tissues and biological fluids at the molecular level, as well as high-density morphological data sets at the tissue and cellular levels. Fifth, instrumentation and methodology development adapt, design, and optimize the 'omics' methodologies required to move applications into the clinical environment. Sixth, bio-engineering generates novel ways to obtain quantitative information from patient samples and develops new methods of delivering and targeting drugs. Finally, information technology and computing databases provide the high-performance computing and high-speed infrastructure required to move, distribute, analyze, archive and manage massive amounts of 'omics' data, and to support the overall process and to implement specific projects.

The capabilities of each and every component of systems medicine are sharply focused on a specific clinical problem. Projects are generated by the clinical teams or disease-focused units and priority should be given to addressing those issues identified by these teams in order to enable the rational design of studies and logical testing of medical intervention and preventive measures. An advantage of applying systems medicine to oncology is that it supports translational research (Supplementary Figure 1) by establishing a constant dynamic two-way dialogue between the clinic and basic science.

Supplementary information in the form of a figure is available on the Nature Clinical Practice Oncology website. 